\title{
Cervical brachytherapy technique for locally advanced carcinoma of the cervix in a patient with septate uterus
}

\author{
Christopher S. Platta, MD!, Charlie Wallace, MS!, Vinai Gondi, MD',2, Rupak Das, PhD', Margaret Straub, PA-Cl, \\ Ahmed Al-Niaimi, MD³, Glenn Applegate, MD4, Kristin A. Bradley, MD' \\ 'Department of Human Oncology, University of Wisconsin Hospital and Clinics, ${ }^{2} \mathrm{CDH}$ Cancer Center, ${ }^{3}$ Department of Gynecologic Oncology. \\ University of Wisconsin Hospital and Clinics, ${ }^{4}$ Turville Bay Radiation Oncology Center, Madison, USA
}

\begin{abstract}
Purpose: To describe an approach to cervical brachytherapy in a patient with congenital septate uterus and locally advanced cervical carcinoma.

Material and methods: The patient is a 34-year-old female with septate uterus presenting with pelvic pain. Workup demonstrated a stage IIB cervical adenocarcinoma with imaging evidence of an involved right external iliac lymph node. The patient received whole pelvic radiation, with concurrent weekly cisplatin $\left(40 \mathrm{mg} / \mathrm{m}^{2}\right)$, to a dose of $45 \mathrm{~Gy}$ in 25 fractions followed by a parametrial boost of $5.4 \mathrm{~Gy}$ and an additional nodal boost of $9 \mathrm{~Gy}$.

Results: The patient was initiated on cervical brachytherapy following fraction 23 of pelvic radiation. To conform to her septated uterus, a Rotte-Y tandem was used. Additionally, 2 CT-compatible ovoids were placed in the vaginal apex to enhance dose distribution and coverage of the target volume. Each fraction of brachytherapy was performed with CT-based planning. A high-risk clinical target volume (HR-CTV) and normal structures were defined and constrained per American Brachytherapy Society (ABS) and Groupe Européen de Curiethérapie/European Society for Therapeutic Radiology and Oncology (GEC-ESTRO) guidelines. The brachytherapy dose was 27.5 Gy in 5 fractions of 5.5 Gy each, prescribed to the HR-CTV.

Conclusions: Herein, we report the first documented case of cervical brachytherapy in a patient with septate uterus and locally advanced cervical carcinoma. Using CT-guided planning, in conjunction with the ABS and GEC-ESTRO guidelines, the patient was effectively treated with adapted cervical brachytherapy, meeting criteria for HR-CTV coverage and normal tissue tolerances.

Key words: brachytherapy, cervical cancer, Rotte-Y tandem, septate uterus.

\section{Purpose}

The standard of care for locally advanced cervical cancer consists of external beam radiation therapy (EBRT) with concurrent cisplatin chemotherapy followed by brachytherapy (BT) boost [1,2]. Until recently, the standard approach for cervical BT consisted of 2D planning with orthogonal X-ray films, prescribing dose to point A. However, with the advent and accessibility of sophisticated imaging systems, cervical BT is now routinely performed with MRI (magnetic resonance imaging) or CT (computed tomography) based planning, prescribing the treatment dose to a clinical target volume (CTV) [3,4]. Either planning method requires an experienced radiation oncologist to ensure proper applicator placement and replicable setup geometry [3]. As a result, the American Brachytherapy Society (ABS) and the Groupe Européen de Curiethérapie/

European Society for Therapeutic Radiology and Oncology (GEC-ESTRO) working group have released specific guidelines to aid radiation oncologists in performing effective and safe cervical BT $[3,4]$.

While anomalies of the uterus and cervix are a relatively rare phenomenon [5], they can significantly impact the ability to perform technically safe and efficacious cervical BT. Additionally, the guidelines set forth by ABS and GEC-ESTRO do not take into account uterine and cervical anatomic anomalies. Given the rarity of female genital tract anomalies, there are only 2 published cases of cervical BT in patients with anatomic uterine abnormalities [6,7]. Herein, we report the unusual case of a patient with a congenital septate uterus, who was subsequently diagnosed with locally advanced adenocarcinoma of the cervix and treated with EBRT, concurrent cisplatin, and CT-guided cervical BT.
\end{abstract}




\section{Material and methods}

\section{Case report: patient history}

The patient is a 34-year-old G4P3 premenopausal female who presented with pain and discomfort during a post-partum pelvic exam in March 2011, 8 weeks after C-section delivery of her third child. She has necessitated a C-section delivery for all of her children due to a congenital septate uterus. Two months post-partum, the patient developed new, progressive dyspareunia with vaginal spotting and clot passage, prompting medical evaluation. Examination by gynecology in December 2011 revealed a large cervical mass which was biopsied, demonstrating poorly differentiated adenocarcinoma consistent with endocervical origin. An endocervical curettage demonstrated poorly differentiated adenocarcinoma and squamous carcinoma in situ, CIN 3, with foci of squamous differentiation within the invasive component of the tumor.

Pretreatment imaging with a pelvic MRI (Fig. 1A) demonstrated a $3.1 \times 2.6 \times 3.0 \mathrm{~cm}$ enhancing mass within the endocervix. Protrusion of the mass was noted into the anterior and posterior vaginal fornices with minimal invasion into uppermost vaginal tissues and bilateral parametrial invasion. There was no evidence of invasion into the bladder or rectum. Also noted was an enlarged node in the right proximal external iliac chain measuring $2.3 \times 1.4 \mathrm{~cm}$. Her septate uterus was radiographically confirmed, with a septate approaching, but not involving, the internal os, making this a Class V uterine anomaly (Table 1) [5].

A positron emission tomography (PET) scan was also obtained and confirmed the presence of a hypermetabolic cervical mass with an SUV max of 15.6. In addition, the enlarged right external iliac lymph node was hypermetabolic with an SUV max of 9.3. No findings of metastatic disease were identified.

In January 2012, the patient was referred to our institution (a tertiary academic center) for further evaluation. On physical examination, she was noted to have a 4 to $4.5 \mathrm{~cm}$ exophytic, friable mass, completely replacing the cervix. Although the tumor was replacing the entire cervix, bimanual exam revealed that the bulk of the tumor was located on the left lateral aspect of the cervix, with palpable disease extension into the posterior and left vaginal fornices. Rectovaginal exam noted left parametrial extension as well. With these exam findings, the patient was staged as International Federation of Gynecology and Obstetrics (FIGO) stage IIB.

The patient was initiated on whole pelvic RT to a dose of $45 \mathrm{~Gy}$ in 25 daily fractions of $1.8 \mathrm{~Gy}$ each using a 4-field technique with $18 \mathrm{MV}$ photons. The bilateral parametria were boosted with an additional dose of 5.4 Gy in 3 fractions. The PET avid right external iliac node received an additional dose of $9 \mathrm{~Gy}$ in 5 fractions, bringing the total dose to the lymph node to $59.4 \mathrm{~Gy}$. Throughout treatment, the patient received concurrent weekly cisplatin chemotherapy at a dose of $40 \mathrm{mg} / \mathrm{m}^{2}$ for 6 cycles.

The patient was evaluated on fraction 18 of EBRT with physical exam and a pre-brachytherapy planning MRI (Fig. 1B). On physical examination, the mass had decreased in size and measured approximately $3 \mathrm{~cm}$, but remained exophytic in nature. Left parametrial involvement was still noted, but improved from initial examination. Magnetic resonance imaging exam demonstrated a $2.3 \times 1.6 \times 1.9 \mathrm{~cm}$ endocervical mass, with partial response to therapy. Additionally, the right external iliac node was decreased in size, now measuring $2.0 \times 1.1 \mathrm{~cm}$, with a central necrotic component.

\section{Brachytherapy technique}

Cervical BT was initiated following fraction 23 of EBRT. The patient was brought to the BT suite where general anesthesia was administered. The patient's septate uterus presented several challenges for BT setup geometry and treatment planning requiring multiple adaptations of customary BT techniques. A standard uterine tandem could not be employed in this setting given the prominent low septate nearly abutting the internal cervical os. To accommodate her septate uterus, a Rotte- $Y$ applicator (Nucletron, an Elekta company, Elekta AB, Stockholm, Sweden) was used (Fig. 2). The Rotte-Y applicator consists of 2 individual uterine tandems that lock together once the applicator is in place. For insertion of the applicator, each uterine septate was first sounded un-

Table 1. American Fertility Society classification of uterine anomalies

\begin{tabular}{lll} 
Class number & Anomaly & Description \\
\hline I & Uterine agenesis/hypoplasia & Uterovaginal agenesis or hypoplasia of variable severity \\
\hline II & Unicornuate uterus & Rudimentary uterine horn with or without a uterine cavity \\
\hline III & Didelphus uterus & $\begin{array}{l}\text { Duplication of the uterus with } 2 \text { distinct uteri and cervices from complete } \\
\text { nonunion of the Müllerian ducts }\end{array}$ \\
\hline IV & Bicornuate uterus & $\begin{array}{l}\text { Presence of myometrial septum extending to the internal or external cervical } \\
\text { os from incomplete union of the Müllerian ducts }\end{array}$ \\
\hline VI & Septate uterus & Uterine septate from failure of complete resorption of uterovaginal septum \\
\hline VII & Arcuate uterus & $\begin{array}{l}\text { Single uterine cavity with a convex or flat fundus from near complete } \\
\text { resorption of the uterovaginal septum }\end{array}$ \\
\hline DES related anomalies & Any uterine anomaly related to DES exposure
\end{tabular}



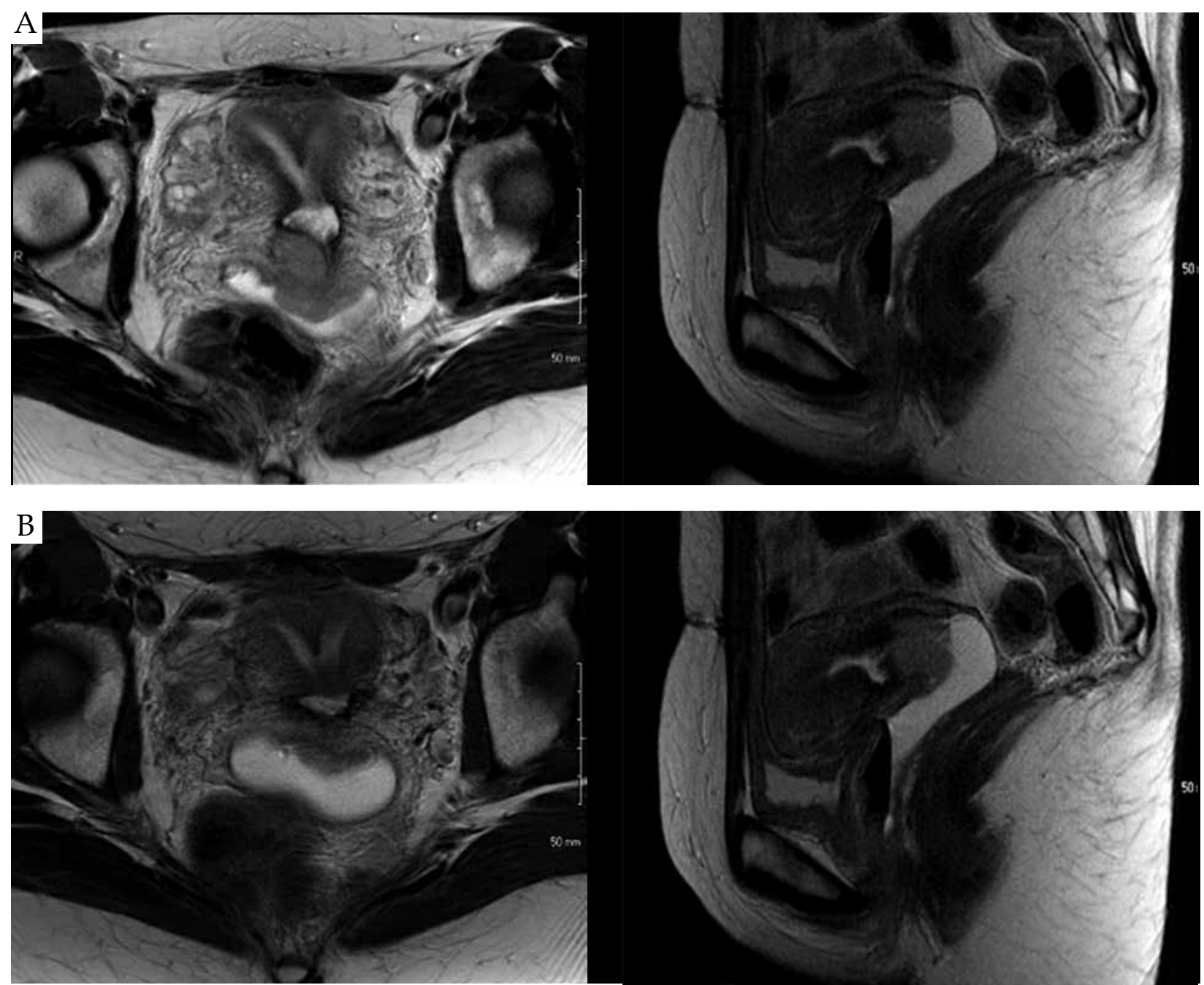

Fig. 1. Pretreatment (A) and pre-brachytherapy (B) MRI images in the axial and sagittal planes demonstrating a septate uterus with a large tumor involving the cervix and parametria. The pre-brachytherapy MRI images were obtained at fraction 18 of whole pelvic radiation with concurrent cisplatin chemotherapy, and demonstrate a partial response to therapy

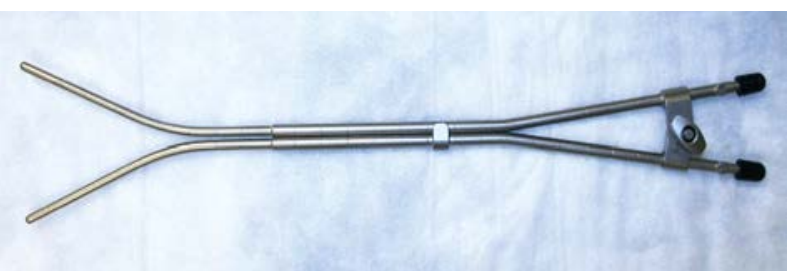

Fig. 2. Photograph of the assembled Rotte-Y applicator prior to placement

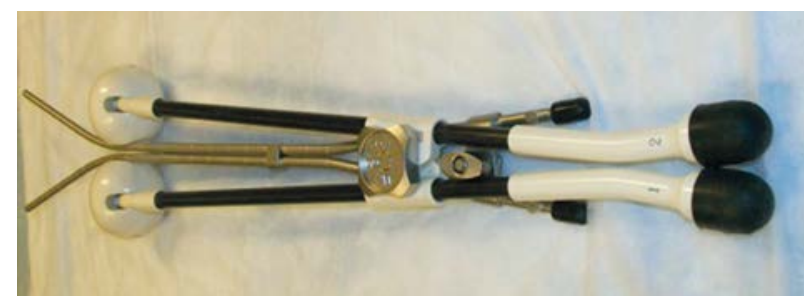

Fig. 3. Photograph of the completely assembled treatment apparatus including the Rotte-Y applicator attached to a pair of colpostats with large ovoids via a simple bridge der ultrasound (US) guidance prior to placement of the tandems. The right tandem was then placed into the right septate with the aid of US. Once the right tandem was in correct position with the tip of the tandem abutting the right fundus, the left tandem was placed in a similar manner. Once the two tandems were in place, they were secured together with the locking mechanism on the external portion of the Rotte-Y apparatus.

After the tandems were successfully placed, two CT-compatible ovoids were assembled onto colpostats (Nucletron, an Elekta company) and placed in the right and left vaginal fornices, respectively, with the pair of tandems bi-secting the ovoids at midplane. A simple bridge was used to connect the Rotte-Y tandem to the colpostats. An example of the completely assembled apparatus can be found in Figure 3. Once the apparatus was secured in place within the uterus and vagina, and the appropriate geometry obtained, extensive vaginal packing was placed into the vagina with $4 \times 4$ gauze to create bladder and rectal separation, as well as stabilize the applicator in a fixed position. 

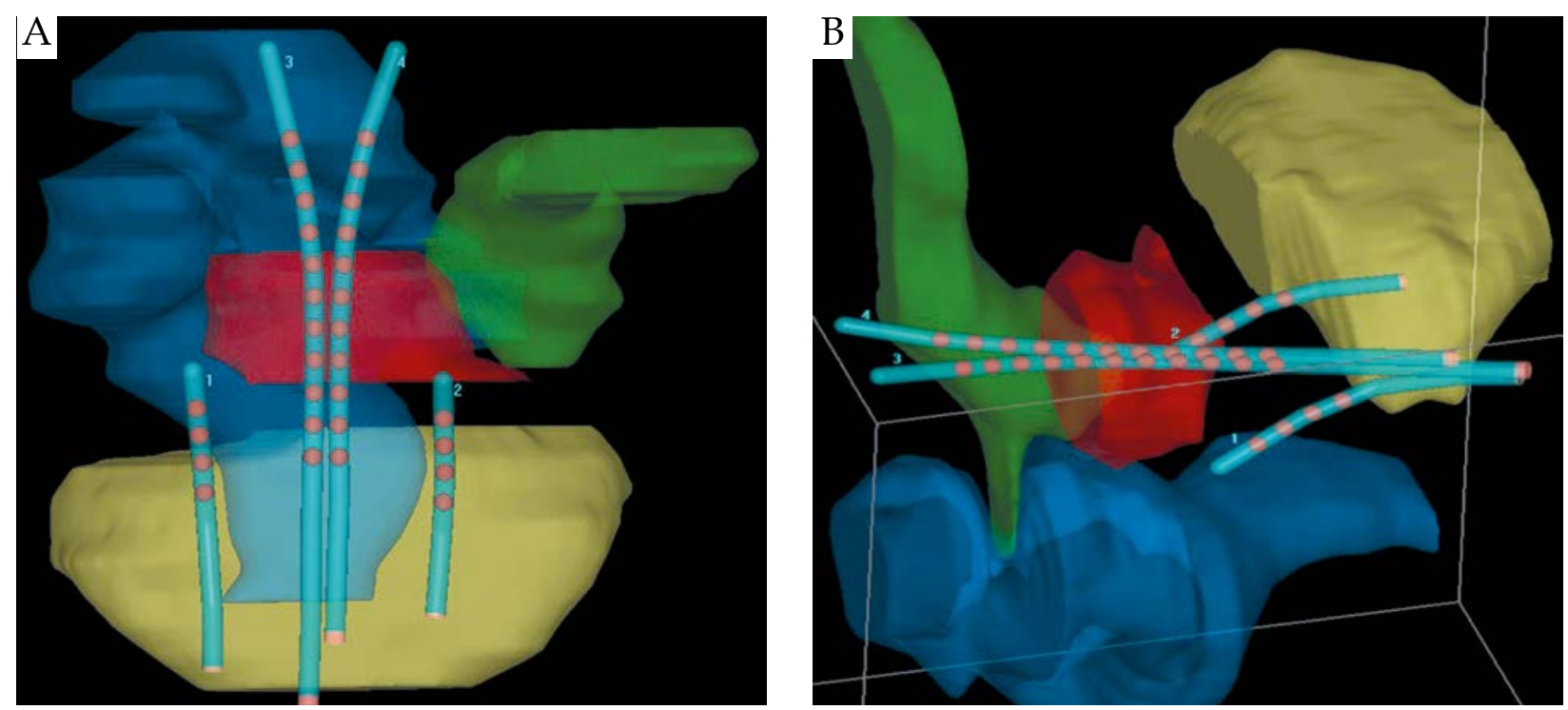

Fig. 4. Three-dimensional graphical rendering of the assembled treatment apparatus in the coronal (A) and right anterior-lateral (B) views, diagraming the anatomic relationship of the apparatus to the HR-CTV (red) and normal structures including the bladder (yellow), rectum (blue), and the sigmoid colon (green). The red dots within the tandems and colpostats represent the planned dwell positions of the HDR ${ }^{192}$ Ir source

The patient was then transferred to the CT simulator to obtain the planning CT images in $2.5 \mathrm{~mm}$ slices. This was performed for each of the five fractions. The CT images were uploaded to the treatment planning system, Oncentra MasterPlan (Nucletron, an Elekta company). The 4 applicator channels ( 2 channels of the Rotte-Y applicator and 2 colpostat channels) were identified and reconstructed on the planning CT. Dwell positions were identified and activated (represented as red dots on the 3D graphical reconstruction found in Figs. 4 and 5). A basic plan was then created as a starting point for further graphical optimization, prescribing a dose of 5.5 Gy to Point $\mathrm{A}$, and a $125 \%$ of the prescription dose (6.875 Gy) to the surface of the ovoids/vaginal mucosa. For purposes of this plan, point A was defined as $2 \mathrm{~cm}$ superior to the line connecting the superior aspect of the ovoids and $2 \mathrm{~cm}$ lateral to the line running between and parallel to the two channels of the Rotte-Y applicator.

Then, using pelvic exam findings and the pre-brachytherapy MRI as a guide, the high risk CTV (HR-CTV) was then contoured per GEC-ESTRO guidelines [4]. The GTV could not be contoured on the CT images given the difficulty with tissue delineation between normal cervix and tumor on the CT. Optimization points were then placed laterally along the length of the Rotte-Y applicator, to deliver the prescription dose superior to point $\mathrm{A}$ and sufficiently cover the HR-CTV. Normal structures including the rectum, sigmoid colon, and bladder were identified and contoured. Figure 4 demonstrates a 3D graphical representation of the applicator and its anatomic relationship to the HR-CTV and normal structures.

Once a computer generated plan was in place, manual optimization of the isodose distribution was then performed on the graphically optimized plan to further enhance HR-CTV coverage and decrease dose to normal structures. Real-time plan analysis was performed taking dose-volume histogram (DVH) data into account to meet normal structure tolerances. A representative coronal image of the 3D plan can be found in Figure 5.

Organ at risk constraints were calculated based on the patient's EBRT dose of $45 \mathrm{~Gy}$ with subsequent parametrial boost (5.4 Gy in 3 fractions), as well as 5 total BT fractions at 5.5 Gy per fraction, per GEC-ESTRO recommendations [8]. Coverage for the HR-CTV was analyzed using the recommend DVH parameter of $\mathrm{D}_{90}$, which was greater than $5.6 \mathrm{~Gy}$ for each fraction of BT. Total $\mathrm{D}_{90}(\mathrm{EBRT}+\mathrm{BT})$ to the HR-CTV was $86.3 \mathrm{~Gy}_{\mathrm{EOD} 2}$. Dose limits to normal structures were within acceptable limits for the entire course of radiation: bladder $\mathrm{D}_{2 \mathrm{cc}}=86.6 \mathrm{~Gy}_{\mathrm{EQD} 2}$, rectum $\mathrm{D}_{2 \mathrm{cc}}=$ $73.7 \mathrm{~Gy}_{\mathrm{EQD} 2}$, and sigmoid $\mathrm{D}_{2 \mathrm{cc}}=72.1 \mathrm{~Gy}_{\mathrm{EQD} 2}$. We were comfortable with the slightly high dose to the rectum and sigmoid, given the final 3 fractions of EBRT were to parametria and lymph node only (above $\mathrm{D}_{2 \mathrm{cc}}$ calculations assumes whole pelvis for all treatments). Treatment was then delivered with high-dose rate (HDR) ${ }^{192} \mathrm{Ir}$ using an HDR after-loading system (Nucletron, an Elekta company). The patient tolerated her BT extremely well, with no significant complications or side effects during treatment. The same radiation oncologist performed all 5 brachytherapy fractions. The total treatment time (EBRT plus BT) was 48 days.

\section{Results}

\section{Patient follow up}

The patient remains without evidence of local or systemic recurrence with a follow up duration of 20 months. A CT scan of the chest, abdomen, and pelvis with contrast at 13 months post treatment was negative. A MRI of the pelvis with and without contrast at 20 months post 


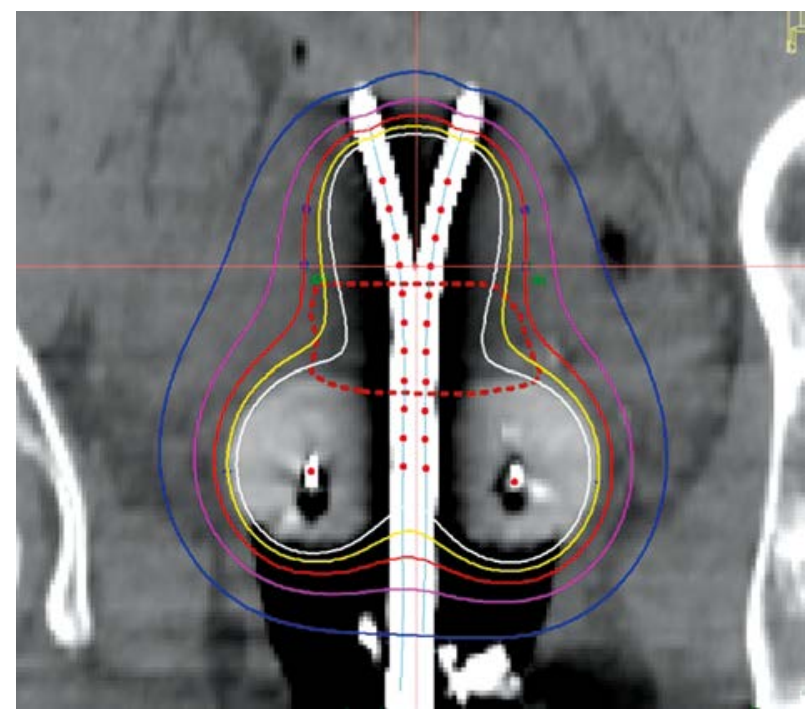

Fig. 5. Representative coronal image from the final brachytherapy plan. The HR-CTV is outlined with a reddashed line. Isodose distribution is demonstrates adequate coverage of the HR-CTV with a conformal brachytherapy plan. The red dots within the tandems and colpostats represent the planned dwell positions of the HDR ${ }^{192}$ Ir source

treatment was also negative for any evidence of recurrence. The patient has received a physical exam with Pap testing at regular 3 months intervals since completing treatment. All exams have been negative for disease recurrence, but have noted mild proximal vaginal narrowing, consistent with a late grade 1 radiation toxicity. All Pap smears have been negative. The patient did report 3 episodes of mild gross hematuria at 19 months post treatment without associated urgency, frequency, or dysuria. Hematuria was confirmed via urinalysis. Cystoscopy demonstrated mild, focal radiation cystitis, consistent with a grade 1 toxicity.

\section{Discussion}

The standard of care for locally advanced cervical carcinoma consists of EBRT with concurrent cisplatin chemotherapy followed by BT boost [1,2]. Multiple studies demonstrate the importance of cervical BT as a component of cervical cancer treatment [9-11]. Furthermore, successful treatment relies heavily on the quality of the implant and implant geometry [3]. Although rare, abnormal uterine anatomy can severely compromise BT geometry if not appropriately recognized prior to the implant. Herein, we report the unusual case of locally advanced adenocarcinoma of the cervix in a patient with a septate uterus, which presented significant challenges in delivering safe and effective cervical BT.

Anatomical anomalies of the female reproductive tract are a rare phenomenon, likely occurring in less than $1 \%$ of females, although the true incidence is a subject to debate with incidence estimates anywhere from $0.16-10 \%$ $[6,12,13]$. Development of the female urogenital tract begins in utero, at approximately week 6 of embryologic life with the Müllerian (paramesonephric) ducts, Wolffian (mesonephric) ducts, and genital ridge all playing critical roles in urogenital tract development. It is aberrant development of the Müllerian ducts which leads to congenital uterine anomalies [6]. These anomalies are most commonly classified according to the American Fertility Society (currently known as the American Society for Reproductive Medicine) classification scheme [5] which breaks down female genital tract anomalies into 7 distinct classes, as outlined in Table 1. This patient has a Class V anomaly: septate uterus resulting from failure of resorption of the septum between two Müllerian ducts.

With the rarity of anatomical uterine anomalies in the general population, the incidence of these patients developing cervical cancer is exceedingly low. However, it has been estimated that as many as $5 \%$ of cervical cancer patients may have undetected malformations. With many cases of cervical cancer diagnosed and treated in economically disadvantaged areas where CT and MRI imaging are not readily available (and as such are not required by FIGO for staging), the true incidence of those with a coexisting uterine anomaly and cervical cancer may be underreported [14]. Most are treated with surgical approaches when feasible, due to anatomic difficulties in managing these patients from a radiotherapeutic perspective [14]. Our review of the literature revealed only 2 cases of cervical cancer in women with congenital anomalies of the female reproductive tract treated with definitive radiotherapy with cervical BT as a component of treatment [6,7].

In 2000, Lee et al. [7] reported a rare case of squamous cell carcinoma of the cervix in a patient with didelphus uterus, a class III Müllerian duct anomaly resulting in two uterine cavities and two cervices. This patient presented with a FIGO stage IIA1 tumor arising from the left cervix, with a minor degree of proximal vaginal involvement. She was treated with whole pelvic EBRT to a dose of $45 \mathrm{~Gy}$ in 25 fractions. This was followed by bilateral cervical BT, placing a tandem into each cervix and respective uterine cavity using a symmetrical dual channel applicator. Orthogonal radiography was used for planning, with the dose prescribed to a modified prescription point $(2 \mathrm{~cm}$ superior to the mean midline position of the two flanges) rather than 2 distinct A points. The use of $2 \mathrm{~A}$ points was not used, as prescribing dose to $2 \mathrm{~cm}$ lateral and $2 \mathrm{~cm}$ superior to each cervical os would have resulted in a much wider prescription isodose surface with the potential for significant overdosing of critical midline structures (bladder, bowel, rectum). The patient was treated with two fractions of HDR, the first prescribed to $6 \mathrm{~Gy}$, the second fraction prescribed to $6.5 \mathrm{~Gy}$.

In 2010, Loo et al. [6] reported a case of stage IIA2 squamous cell carcinoma of the cervix with bicornuate bicollis uterus (class IV Müllerian duct anomaly), treated with whole pelvic EBRT and bilateral intracavitary BT. This patient received EBRT to a dose of 50 Gy in 25 fractions with weekly cisplatin chemotherapy at a dose of $40 \mathrm{mg} / \mathrm{m}^{2}$. Intracavitary BT was performed using low dose rate (LDR) techniques with ${ }^{137} \mathrm{Cs}$ using a Perspex vaginal cylinder applicator with a tungsten rectal shield. The patient was treated to a dose of 18 Gy over 2 fractions prescribed to Manchester point A. On the first fraction 
of BT, the right side was treated with a calculation also being preformed to the left point $\mathrm{A}$ with the aid of a wire marker in the left uterine cavity. For the second fraction of treatment, the geometry was reversed so that the left side was treated and the dose to the right point $\mathrm{A}$ was calculated using the wire marked in the right uterine cavity.

Our review of the literature did not find any previous report of a patient with a septate uterus treated with cervical BT. Here, we report the first published case of cervical carcinoma in a patient with a septate uterus managed with concurrent chemo and radiation therapy. Additionally, this is the first paper in the literature to describe the process of cervical BT in a patient with a congenital uterine anomaly using CT-based planning with adaptation of the GEC-ESTRO and ABS guidelines.

Like Lee et al. [7], we used a symmetric dual channel applicator, with separate tandems placed into the right and left uterine septate, respectively. Unlike the patient described by Lee et al. [7], our patient had one cervix, making the setup geometry slightly less complex. Our patient also had large vaginal fornices, allowing the placement of CT-compatible ovoids in the vaginal apex, aiding in our treatment planning, geometry, and target volume coverage, allowing for increased dose conformity to the HR-CTV. Neither Lee et al. [7] or Loo et al. [6] utilized ovoids in their documented treatment approaches.

Unlike Lee et al. [7] and Loo et al. [6], who used orthogonal imaging for planning, we utilized CT based planning for each fraction. Ideally, MRI based planning would be used in such a delicate case. However, this was not possible as the Rotte-Y applicator was not MRI compatible. MRI planning has notably demonstrated advantages in the setting of classic cervical brachytherapy [15].

Three-dimensional planning techniques were undoubtedly useful in accurately delineating the target volume (HR-CTV), and reducing dose to normal structures via manual optimization of isodose distribution on the treatment planning system in this patient. Critical analysis of HR-CTV coverage and dose to critical organs could be analyzed and adjusted based on readily available DVH data, which is not possible using 2D planning techniques.

With the adaptation of sophisticated imaging systems such as CT and MRI, the ability to perform cervical BT with greater accuracy continues to improve. In the setting of uterine anomalies, CT or MRI guided planning can greatly improve the ability to deliver safe and effective BT with improved target delineation and coverage, while minimizing dose to adjacent normal tissues. The application of ABS and GEC-ESTRO guidelines are helpful in patients with uterine anomalies treated with 3D-based cervical BT as a guide for delineating and monitoring coverage of, and doses to, the target volume are nearby normal structures.

\section{Disclosure}

Vinai Gondi has served on the Medical Advisory Board for Novocure and has received an honorarium from the American Board of Medical Specialties (ABMS). The remaining authors have no conflicts of interest to report with regard to publication of this manuscript.

\section{Disclosure}

Authors report no conflict of interest.

\section{References}

1. Eifel PJ, Winter K, Morris $M$ et al. Pelvic irradiation with concurrent chemotherapy versus pelvic and para-aortic irradiation for high-risk cervical cancer: an update of radiation therapy oncology group trial (RTOG) 90-01. J Clin Oncol 2004; 22: $872-880$.

2. Rose PG, Bundy BN, Watkins EB et al. Concurrent cisplatin-based radiotherapy and chemotherapy for locally advanced cervical cancer. N Engl J Med 1999; 340: 1144-1153.

3. Viswanathan AN, Thomadsen B. American Brachytherapy Society consensus guidelines for locally advanced carcinoma of the cervix. Part I: general principles. Brachytherapy 2012; 11: 33-46.

4. Haie-Meder C, Pötter R, Van Limbergen E et al. Recommendations from Gynaecological (GYN) GEC-ESTRO Working Group (I): concepts and terms in 3D image based 3D treatment planning in cervix cancer brachytherapy with emphasis on MRI assessment of GTV and CTV. Radiother Oncol 2005; 74: 235-245.

5. The American Fertility Society classifications of adnexal adhesions, distal tubal occlusion, tubal occlusion secondary to tubal ligation, tubal pregnancies, mullerian anomalies and intrauterine adhesions. Fertil Steril 1988; 49: 944-955.

6. Loo HW, Locks SM. Squamo us cell carcinoma of the cervix: report of an unusual case of bicornuate bicollis uterus treated with bilateral intracavity brachytherapy. Br J Radiol 2010; 83: e143-146.

7. Lee CD, Churn M, Haddad $\mathrm{N}$ et al. Bilateral radical radiotherapy in a patient with uterus didelphys. Br J Radiol 2000; 73: 553-556.

8. Pötter R, Haie-Meder C, Van Limbergen E et al. Recommendations from gynaecological (GYN) GEC ESTRO working group (II): concepts and terms in 3D image-based treatment planning in cervix cancer brachytherapy-3D dose volume parameters and aspects of 3D image-based anatomy, radiation physics, radiobiology. Radiother Oncol 2006; 78: 67-77.

9. Lanciano RM, Won M, Coia LR, Hanks GE. Pretreatment and treatment factors associated with improved outcome in squamous cell carcinoma of the uterine cervix: a final report of the 1973 and 1978 patterns of care studies. Int J Radiat Oncol Biol Phys 1991; 20: 667-676.

10. Hanks GE, Herring DF, Kramer S. Patterns of care outcome studies. Results of the national practice in cancer of the cervix. Cancer 1983; 51: 959-967.

11. Coia L, Won M, Lanciano R et al. The Patterns of Care Outcome Study for cancer of the uterine cervix. Results of the Second National Practice Survey. Cancer 1990; 66: 2451-2456.

12. Acién P, Acién M, Sánchez-Ferrer M. Complex malformations of the female genital tract. New types and revision of classification. Hum Reprod 2004; 19: 2377-2384.

13. Troiano RN, McCarthy SM. Mullerian duct anomalies: imaging and clinical issues. Radiology 2004; 223: 19-34.

14. Rastogi M, Revannasiddaiah S, Thakur P et al. Mullerian duct anomalies and their effect on the radiotherapeutic management of cervical cancer. Chin J Cancer 2013; 32: 434-40.

15. Anderson JW, Xia J, Flynn RT et al. High resolution (3 Tesla) MRI-guided conformal brachytherapy for cervical cancer: consequences of different high-rick CTV sizes. J Contemp Brachytherapy 2013; 5: 101-109. 